\title{
Rates, Predictors and Variability of Interhospital Transfers: A National Evaluation
}

\author{
Stephanie K. Mueller, MD, MPH', ${ }^{1 *}$, Jie Zheng ${ }^{3}$, E. John Orav, PhD ${ }^{1,3}$, Jeffrey L. Schnipper, MD, MPH ${ }^{1,2}$
}

'Division of General Internal Medicine, Brigham and Women's Hospital, Boston, Massachusetts; 'Harvard Medical School, Boston, Massachusetts; ${ }^{3}$ Harvard T.H. Chan School of Public Health, Boston, Massachusetts.

\begin{abstract}
IMPORTANCE: Interhospital transfer (IHT) remains a largely unstudied process of care.
\end{abstract}

OBJECTIVE: To determine the nationwide frequency of, patient and hospital-level predictors of, and hospital variability in IHT.

DESIGN: Cross-sectional study.

SETTING: Centers for Medicare and Medicaid 2013 100\% Master Beneficiary Summary and Inpatient claims files merged with 2013 American Hospital Association data.

PATIENTS: Beneficiaries $\geq 65$ years and older enrolled in Medicare $A$ and $B$, with an acute care hospitalization claim in 2013.

EXPOSURES: Patient and hospital characteristics of transferred and nontransferred patients.

MEASUREMENTS: Frequency of interhospital transfers (IHT); adjusted odds of transfer of each patient and each hospital characteristic; and variability in hospital transfer rates.

RESULTS: Of 6.6 million eligible beneficiaries with an acute care hospitalization, 101,507 (1.5\%) underwent IHT. Selected characteristics associated with greater adjusted odds of transfer included: patient age 74-85 years (odds ratio [OR], 2.38 compared with $65-74$ years; 95\% confidence intervals [Cl], 2.33-2.43); nonblack race (OR, 1.17; 95\% Cl, 1.13-1.20); higher comorbidity (OR, 1.37; 95\% Cl, 1.36-1.37); lower diagnosisrelated group-weight (OR, 2.02; 95\% Cl, 1.95-2.09); fewer recent hospitalizations (OR, 1.87; 95\% Cl, 1.79-1.95); and hospitalization in the Northeast (OR, 1.40; 95\% Cl, 1.27-1.55). Higher case mix index of the hospital was associated with a lower adjusted odds of transfer (OR, 0.36; 95\% Cl, 0.30-0.45). Variability in hospital transfer rates remained significant after adjustment for patient and hospital characteristics (variance $0.28, P=0.01$ ).

CONCLUSIONS: In this nationally representative evaluation, we found that a sizable number of patients undergo IHT. We identified both expected and unexpected patient and hospital-level predictors of IHT, as well as unexplained variability in hospital transfer rates, suggesting lack of standardization of this complex care transition. Our study highlights further investigative avenues to help guide best practices in IHT. Journal of Hospital Medicine 2017;12:435-442. (C) 2017 Society of Hospital Medicine
Interhospital transfer (IHT) is defined as the transfer of hospitalized patients between acute care hospitals. Although cited reasons for transfer include providing patients access to unique specialty services, ${ }^{1}$ patterns and practices of IHT remain largely unstudied. Interhospital transfer is known to be common in certain patient populations, including selected patients presenting to the intensive care unit ${ }^{2}$ and those with acute myocardial infarction (AMI), ${ }^{3-5}$ but no recent studies have looked at frequency of IHT among a broader group of hospitalized patients nationally. Little is known about which patients are selected for transfer and why. ${ }^{6}$ Limited evidence suggests poor concordance between cited reason for transfer among patients, transferring physicians, and receiving physicians, ${ }^{7}$ indicating ambiguity in this care process.

\footnotetext{
*Address for correspondence and reprint requests: Stephanie K. Mueller, MD, MPH, Division of General Internal Medicine, Brigham and Women's Hospital, 1620 Tremont Street, Roxbury, MA 02120; Telephone: 617-278-0628; Fax: 617-732-7072; E-mail: smueller1@partners.org

Additional Supporting Information may be found in the online version of this article.

Received: December 7, 2016; Revised: February 13, 2017; Accepted: February 23, 2017
}

2017 Society of Hospital Medicine DOI 10.12788/jhm.2747
Interhospital transfer exposes patients to the potential risks associated with discontinuity of care. Communication is particularly vulnerable to error during times of transition. ${ }^{8-10}$ Patients transferred between acute care hospitals are especially vulnerable, given the severity of illness in this patient population, ${ }^{11}$ and the absence of other factors to fill in gaps in communication, such as common electronic health records. Limited existing literature suggests transferred patients use more resources ${ }^{12-13}$ and experience worse outcomes compared to nontransferred patients, ${ }^{11}$ although these data involved limited patient populations, and adjustment for illness severity and other factors was variably addressed. ${ }^{14-16}$

To improve the quality and safety of IHT, therefore, it is necessary to understand which patients benefit from IHT and identify best practices in the IHT process. ${ }^{17} \mathrm{~A}$ fundamental first step is to study patterns and practices of IHT, in particular with an eye towards identifying unwarranted variation. ${ }^{18}$ This is important to understand the prevalence of the issue, provide possible evidence of lack of standardization, and natural experiments with which to identify best practices.

To address this, we conducted a foundational study examining a national sample of Medicare patients to determine the nationwide frequency of IHT among elderly patients, 
patient and hospital-level predictors of transfer, and hospital variability in IHT practices.

\section{METHODS}

We performed a cross-sectional analysis using 2 nationally representative datasets: (1) Center for Medicare and Medicaid Services (CMS) 2013 100\% Master Beneficiary Summary and Inpatient claims files, which contains data on all fee-for-service program Medicare enrollees' demographic information, date of death, and hospitalization claims, including ICD-9 codes for diagnoses, diagnosis-related group (DRG), and dates of service; merged with (2) 2013 American Hospital Association (AHA) data, ${ }^{19}$ which contains hospital-level characteristics for all acute care hospitals in the U.S. Our study protocol was approved by the Partners Healthcare Human Subjects Review Committee.

Beneficiaries were eligible for inclusion if they were 65 years or older, continuously enrolled in Medicare A and B, with an acute care hospitalization claim in 2013, excluding Medicare managed care and end-stage renal disease (ESRD) beneficiaries. We additionally excluded beneficiaries hospitalized at federal or nonacute care hospitals, or critical access hospitals given their mission to stabilize and transfer patients to referral hospitals. ${ }^{20}$

Transferred patients were defined as: (1) beneficiaries with a "transfer out" claim and a corresponding "transfer in" claim at a different hospital; as well as (2) beneficiaries with a "transfer out" claim and a corresponding date of admission to another hospital within 1 day following the date of claim; and (3) beneficiaries with a "transfer in" claim and a corresponding date of discharge from another hospital within 1 day preceding the date of claim. Beneficiaries transferred to the same hospital, or cared for at hospitals with "outlier" transfer in rates equal to $100 \%$ or transfer out rates greater than $35 \%$, were excluded from analysis given the suggestion of nonstandard claims practices. Beneficiaries with greater than 1 transfer within the same hospitalization were additionally excluded.

\section{Patient Characteristics}

Patient characteristics were obtained from the CMS data files and included: demographics (age, sex, race); DRGweight, categorized into quartiles; primary diagnosis for the index hospitalization using ICD-9 codes; patient comorbidity using ICD-9 codes compiled into a CMS-Hierarchical Condition Category (HCC) risk score; ${ }^{21}$ presence of Medicaid co-insurance; number of hospitalizations in the past 12 months, categorized into 0, 1, 2-3, and 4 or more; season, defined as calendar quarters; and median income per household by census tract. These characteristics were chosen a priori given expert opinion in combination with prior research demonstrating association with IHT. ${ }^{11,22}$

\section{Hospital Characteristics}

Hospital characteristics were obtained from AHA data files and included hospitals' size, categorized into small, medium, and large (less than 100, 100 to 399, 400 or more beds); geographic location; ownership; teaching status; setting (urban vs. rural); case mix index (CMI) for all patients cared for at the hospital; and presence of selected specialty services, including certified trauma center, medical intensive care unit, cardiac intensive care unit, cardiac surgery services, adult interventional cardiac catheterization, adult cardiac electrophysiology, and composite score of presence of 55 other specialty services (complete list in Appendix A). All characteristics were chosen a priori given expert opinion or relationship of characteristics with IHT, and prior research utilizing AHA data. ${ }^{23-24}$

\section{Analysis}

Descriptive statistics were used to evaluate the frequency of IHT, characteristics of transferred patients, and number of days to transfer. Patient and hospital characteristics of transferred vs. nontransferred patients were compared using chisquare analyses.

To analyze the effects of each patient and hospital characteristic on the odds of transfer, we used logistic regression models incorporating all patient and hospital characteristics, accounting for fixed effects for diagnosis, and utilizing generalized estimating equations (the GENMOD procedure in SAS statistical software, v 9.4; SAS Institute Inc., Cary, North Carolina) to account for the clustering of patients within hospitals. ${ }^{25}$ Indicator variables were created for missing covariate data and included in analyses when missing data accounted for greater than $10 \%$ of the total cohort.

To measure the variability in transfer rates between hospitals, we used a sequence of random effects logistic regression models. We first ran a model with no covariates, representing the unadjusted differences in transfer rates between hospitals. We then added patient characteristics to see if the unadjusted differences in IHT rates were explained by differences in patient characteristics between hospitals. Lastly, we added hospital characteristics to determine if these explained the remaining differences in transfer rates. Each of the 3 models provided a measure of between-hospital variability, reflecting the degree to which IHT rates differed between hospitals. Additionally, we used the intercept from the unadjusted model and the measure of between-hospital variability from each model to calculate the $95 \%$ confidence intervals, illustrating the range of IHT rates spanning 95\% of all hospitals. We used those same numbers to calculate the $25^{\text {th }}$ and $75^{\text {th }}$ percentiles, illustrating the range of IHT rates for the middle half of hospitals.

\section{RESULTS}

Among 28 million eligible beneficiaries, 6.6 million had an acute care hospitalization to nonfederal, noncritical access hospitals, and 107,741 met our defined criteria for IHT. An additional 3790 beneficiaries were excluded for being transferred to the same facility, 416 beneficiaries (115 transferred, 301 nontransferred) were excluded as they were cared for at 1 of the 11 hospitals with "outlier" transfer in/out rates, and 


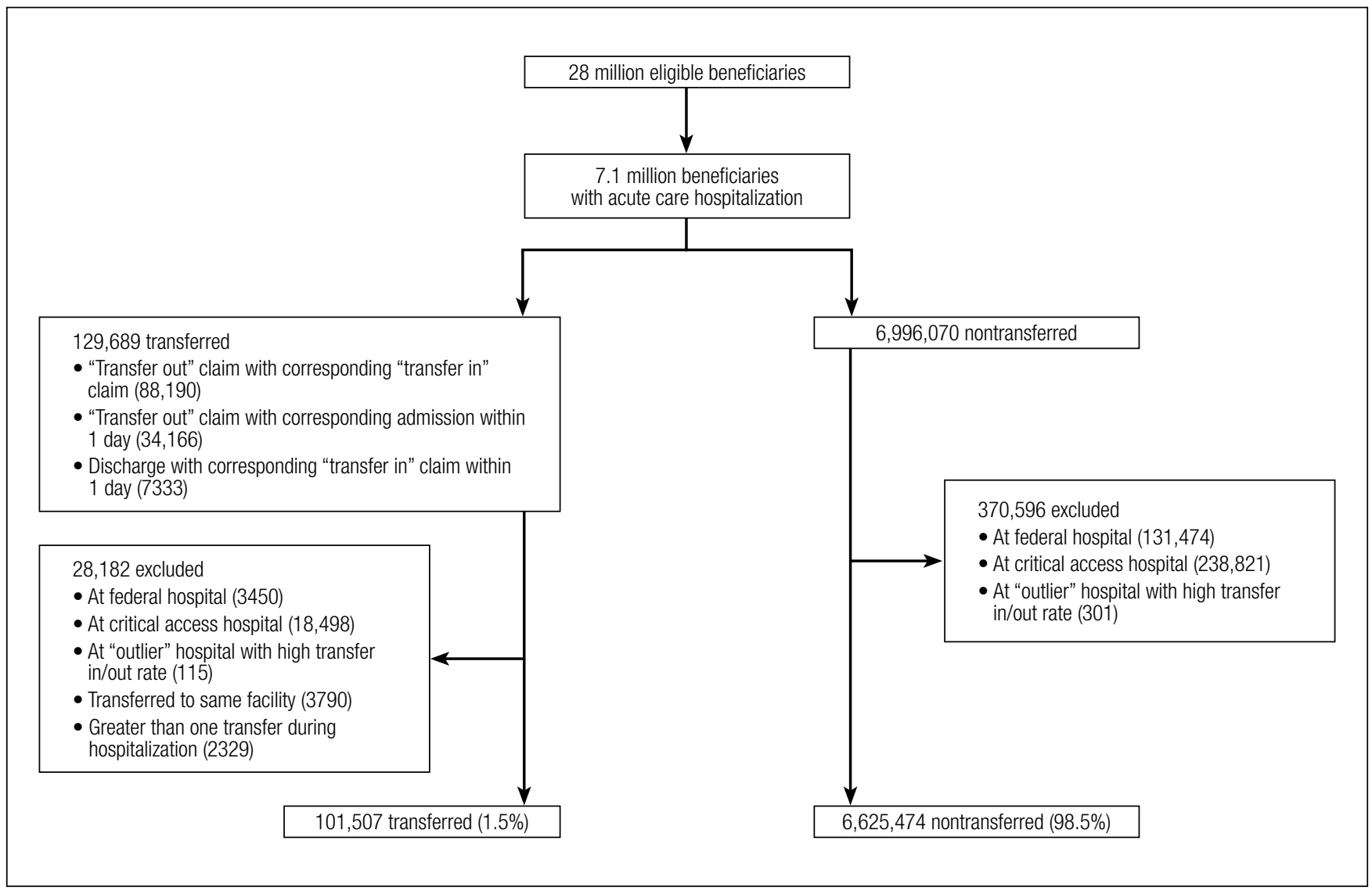

FIG. 1. Cohort selection. ${ }^{a}$

${ }^{a}$ Centers for Medicare and Medicaid Services 2013 100\% Master Beneficiary Summary and Inpatient claims files.

2329 were excluded because they had more than 1 transfer during hospitalization. Thus, the final cohort consisted of 101,507 transferred $(1.5 \%)$ and 6,625,474 nontransferred beneficiaries (Figure 1). Of the 101,507 transferred beneficiaries, 2799 (2.8\%) were included more than once (ie, experienced more than 1 IHT on separate hospitalizations throughout the study period; the vast majority of these had 2 separate hospitalizations resulting in IHT). Characteristics of transferred and nontransferred beneficiaries are shown (Table 1).

Among transferred patients, the top 5 primary diagnoses at time of transfer included AMI (12.2\%), congestive heart failure (CHF) (7.2\%), sepsis (6.6\%), arrhythmia (6.6\%), and pneumonia (3.4\%). Comorbid conditions most commonly present in transferred patients included CHF (52.6\%), renal failure $(51.8 \%)$, arrhythmia $(49.8 \%)$, and chronic obstructive pulmonary disease (COPD; $37.0 \%$ ). The most common day of transfer was day after admission (hospital day 2, $24.7 \%$ ), with $75 \%$ of transferred patients transferred before hospital day 6 (Appendix B).

After adjusting for all other patient and hospital characteristics and clustering by hospital, the following variables were associated with greater odds of transfer: older age, male sex, nonblack race, non-Medicaid co-insurance, higher comorbidity (HCC score), lower DRG-weight, and fewer hos-

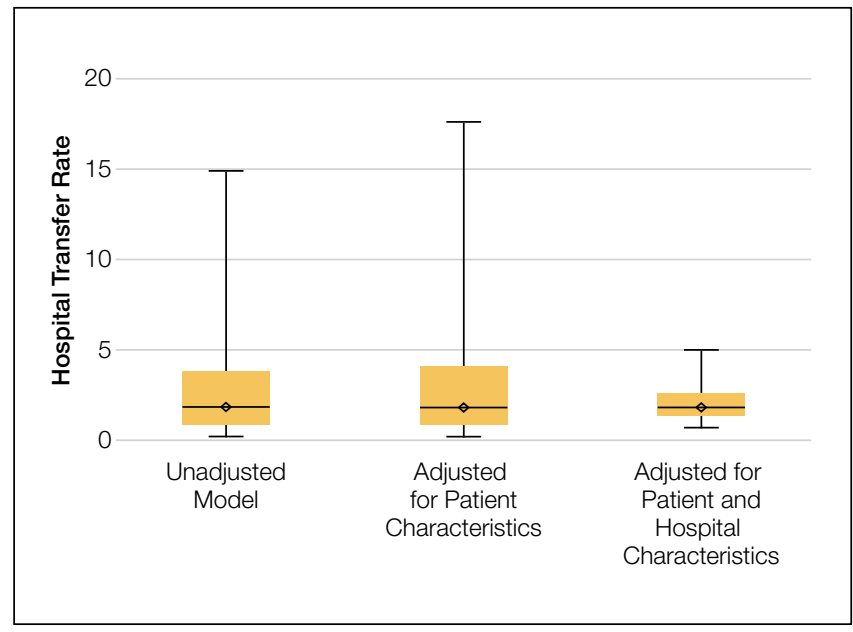

FIG. 2. Distribution of transfer rates across hospitals.

NOTE: All models are centered at the median transfer rate of $1.79 \%$. Shaded boxes encompass transfer rates from the 25th percentile (Q3) for each model. Whiskers encompass transfer rates from the 2.5th percentile to the 97.5 th percentile. For example, the null model demonstrates that half of all hospitals have transfer rates between $\mathrm{Q} 1=0.83 \%$ and $\mathrm{Q} 3=3.80 \%$; after equalizing the patient characteristics, the interquartile ranges expands to $\mathrm{Q} 1$ $=0.78 \%$ to $Q 3=4.06 \%$; however, measured hospital characteristics explain most of this variability, reducing the interquartile range to $\mathrm{Q} 1=1.26 \%$ to $\mathrm{Q} 3=2.54 \%$.

pitalizations in the prior 12 months. Beneficiaries also had greater odds of transfer if initially hospitalized at smaller hospitals, nonteaching hospitals, public hospitals, at hospitals in the Northeast, those with fewer specialty services, and 
TABLE 1. Baseline Characteristics of Transferred vs. Nontransferred Beneficiaries

\begin{tabular}{|c|c|c|c|}
\hline Characteristic & $\begin{array}{l}\text { Transferred } \\
(n=101,507)\end{array}$ & $\begin{array}{l}\text { Nontransferred } \\
(n=6,625,474)\end{array}$ & $P$ value \\
\hline \multicolumn{4}{|l|}{ Patient Characteristics } \\
\hline \multicolumn{4}{|l|}{ Age, $n(\%)$} \\
\hline $65-74$ & $42,245(41.6)$ & $2,328,830(35.1)$ & \multirow{3}{*}{$<0.001$} \\
\hline $75-84$ & $40,630(40.0)$ & $2,419,802(36.5)$ & \\
\hline$\geq 85$ & $18,632(18.4)$ & $1,876,842(28.3)$ & \\
\hline Male, n (\%) & $49,830(49.1)$ & $2,800,503(42.3)$ & $<0.001$ \\
\hline \multicolumn{4}{|l|}{ Race, n (\%) } \\
\hline White & $88,873(87.6)$ & $5,711,376(86.2)$ & \multirow{4}{*}{$<0.001$} \\
\hline Black & $8,381(8.3)$ & $596,347(9.0)$ & \\
\hline Hispanic & $1,273(1.3)$ & $112,580(1.7)$ & \\
\hline Other & $2,980(2.9)$ & $205,171(3.1)$ & \\
\hline \multicolumn{4}{|l|}{ DRG-weight quartile, n (\%) } \\
\hline Lowest quartile & $29,883(29.4)$ & $1,669,620(25.2)$ & \multirow{4}{*}{$<0.001$} \\
\hline 2nd quartile & $28,007(27.6)$ & $1,629,864(24.6)$ & \\
\hline 3rd quartile & 28,992(28.6) & $1,696,125(25.6)$ & \\
\hline Highest quartile & $14,625(14.4)$ & $1,629,865(24.6)$ & \\
\hline \multicolumn{4}{|l|}{ Primary diagnosis on admission, $\mathrm{n}(\%)$} \\
\hline AMI & $12,395(12.2)$ & $172,845(2.6)$ & \multirow{16}{*}{$<0.001$} \\
\hline CHF & $7,341(7.2)$ & $379,372(5.7)$ & \\
\hline Sepsis & $6,682(6.6)$ & $419,110(6.4)$ & \\
\hline Arrhythmia & $6,687(6.6)$ & $300,126(4.6)$ & \\
\hline Stroke & $3,640(3.6)$ & $211,593(3.1)$ & \\
\hline Pneumonia & $3,461(3.4)$ & $300,804(4.6)$ & \\
\hline Gl bleed & $3,089(3.0)$ & $178,606(2.7)$ & \\
\hline Renal failure & $2,085(2.1)$ & $188,021(2.8)$ & \\
\hline Esophageal & $1,948(1.9)$ & $230,289(3.4)$ & \\
\hline COPD & $1,809(1.8)$ & $258,984(3.8)$ & \\
\hline Hip fracture/dislocation & $1,690(1.7)$ & $158,915(2.4)$ & \\
\hline Chest pain & $896(0.9)$ & $66,288(1.0)$ & \\
\hline UTI & $924(0.9)$ & $414,999(6.3)$ & \\
\hline Respiratory disease & 799(0.8) & $84,180(1.3)$ & \\
\hline Metabolic & $845(0.8)$ & $121,321(1.8)$ & \\
\hline Other & $47,216(46.5)$ & $3,140,021(47.5)$ & \\
\hline HCC risk score, mean (SD) ${ }^{\mathrm{a}}$ & $3.5(2.0)$ & $2.6(1.8)$ & $<0.001$ \\
\hline \multicolumn{4}{|l|}{ Top comorbid conditions, $\mathrm{n}(\%)$} \\
\hline $\mathrm{CHF}$ & $53,397(52.6)$ & $2,383,413(36.0)$ & \multirow{4}{*}{$<0.001$} \\
\hline Renal failure & $52,542(51.8)$ & $2,599,411(39.2)$ & \\
\hline Arrhythmia & $50,577(49.8)$ & $2,363,757(35.7)$ & \\
\hline COPD & $37,511(37.0)$ & $2,014,789(30.4)$ & \\
\hline Medicaid co-insurance, $\mathrm{n}(\%)$ & $19,326(19.0)$ & $1,337,310(20.2)$ & $<0.001$ \\
\hline \multicolumn{4}{|l|}{ Number of hospitalizations in the past 12 months, $n$ (\%) } \\
\hline 0 & $67,944(66.9)$ & $4,296,542(64.8)$ & \multirow{4}{*}{$<0.001$} \\
\hline 1 & $18,748(18.5)$ & $1,336,788(20.2)$ & \\
\hline $2-3$ & $12,382(12.2)$ & $843,101(12.7)$ & \\
\hline$\geq 4$ & $2,433(2.4)$ & $149,043(2.3)$ & \\
\hline \multicolumn{4}{|l|}{ Season of hospital admission ${ }^{b}$} \\
\hline Q1 & $27,148(26.7)$ & $1,797,723(27.1)$ & \multirow{4}{*}{$<0.001$} \\
\hline Q2 & $26,153(25.8)$ & $1,658,024(25.0)$ & \\
\hline Q3 & $25,317(24.9)$ & $1,581,828(23.9)$ & \\
\hline Q4 & $22,889(22.5)$ & $1,587,899(24.0)$ & \\
\hline Median income per household by census tract, mean (SD) & $52,818.5(21,932)$ & $53,241.3(23,272)$ & $<0.001$ \\
\hline \multicolumn{4}{|l|}{ Index Hospital Characteristics ${ }^{\circ}$} \\
\hline \multicolumn{4}{|l|}{ Size, n (\%) } \\
\hline Small (<99 beds) & $27,422(27.0)$ & $561,838(8.5)$ & \multirow{3}{*}{$<0.001$} \\
\hline Medium (100-399 beds) & $62,307(61.4)$ & $3,743,514(56.5)$ & \\
\hline Large ( $\geq 400$ beds) & $11,778(11.6)$ & $2,320,122(35.0)$ & \\
\hline
\end{tabular}

Continued on page 439 those with a low CMI (Table 2).

In examining the between-hospital variability in IHT, our unadjusted model estimated an average transfer rate of $1.79 \%$, and showed a variance estimate of $1.33(P=0.009)$, demonstrating that $95 \%$ of hospitals have transfer rates between $0.83 \%$ and $3.80 \%$. The variance estimate increased by $19 \%$ to $1.58(P=0.009)$ when adjusting for patient characteristics. After adjusting for hospital characteristics, variance decreased by $83 \%$ to 0.28 $(P=0.01)$, showing $95 \%$ of hospitals have transfer rates between $1.26 \%$ and $2.54 \%$ (Figure 2 ).

\section{DISCUSSION}

In this nationally representative study of $6.6 \mathrm{mil}$ lion Medicare beneficiaries, we found that 1.5\% of patients were transferred between acute care facilities and were most often transferred prior to hospital day 6. Older age, male sex, nonblack race, higher medical comorbidity, lower DRG weight, and fewer recent hospitalizations were associated with greater odds of transfer. Initial hospitalization at smaller, nonteaching, public hospitals, with fewer specialty services were associated with greater odds of transfer, while higher CMI was associated with a lower odds of transfer. The most common comorbid conditions among transferred patients included CHF, renal failure, arrhythmia, and COPD; particularly notable was the very high prevalence of these conditions among transferred as compared with nontransferred patients. Importantly, we found significant variation in IHT by region and a large variation in transfer practices by hospital, with significant variability in transfer rates even after accounting for known patient and hospital characteristics.

Among our examined population, we found that a sizable number of patients undergo IHT-more than 100,000 per year. Primary diagnoses at time of transfer consist of common inpatient conditions, including AMI, CHF, sepsis, arrhythmia, and pneumonia. Limited prior data support our findings, with up to $50 \%$ of AMI patients reportedly undergoing $\mathrm{IHT}^{3-5}$ and severe sepsis and respiratory illness reported as common diagnoses at transfer. ${ }^{11}$ Although knowledge of these primary diagnoses does not directly confer an understanding of reason for transfer, one can speculate based on our findings. For example, research demonstrates the majority of AMI patients who undergo IHT had further intervention, including stress testing, cardiac catheterization, and/or coronary artery bypass graft surgery. ${ }^{5,26}$ Thus, it is reasonable to presume that many of the beneficiaries transferred with AMI were transferred to receive this more specialized cardiac care. We further found the majority of patients are transferred 
prior to hospital day 6 with the highest prevalence on day 2 , supporting the hypothesis that these patients may be transferred for receipt of specialty services for their admission diagnosis. However, we cannot prove this presumption, and for other conditions, such as pneumonia, the plan after IHT is less obvious. There are numerous possible reasons for transfer, ${ }^{1}$ including patient preference and prior affiliation with receiving hospital. Further research is required to more fully define these reasons in greater detail.

We additionally found that certain patient characteristics were associated with greater odds of transfer. Research suggests that transferred patients are "sicker" than nontransferred patients. ${ }^{1,11}$ Although our findings in part confirm these data, we paradoxically found that higher DRG-weight and 4 or more hospitalizations in the past year were actually associated with lower odds of transfer. In addition, the oldest patients in our cohort $(85$ years or older) were actually less likely to be transferred than their slightly younger counterparts ( 75 to 84 years). These variables may reflect extreme illness or frailty, ${ }^{27}$ and providers consciously (or subconsciously) may factor this in to their decision to transfer, considering a threshold past which transfer would confer more risk than benefit (eg, a patient may be "too sick" for transfer). Indeed, in a secondary analysis without hospital characteristics or comorbidities, and with fixed effects by hospital, we found the highest rates of IHT in patients in the middle 2 quartiles of DRG-weight, supporting this threshold hypothesis. It is also possible that patients with numerous hospitalizations may be less likely to be transferred because of familiarity and a strong sense of responsibility to continue to care for those patients (although we cannot confirm that those prior hospitalizations were all with the same index hospital).

It is also notable that odds of transfer differed by race, with black patients $17 \%$ less likely to undergo transfer compared to whites, similar to findings in other IHT studies. ${ }^{11}$ This finding, in combination with our demonstration that Medicaid patients also have lower odds of transfer, warrants further investigation to ensure the process of IHT does not bias against these populations, as with other well-documented health disparities..$^{28-30}$

The hospital predictors of transfer were largely expected. However, interestingly, when we controlled for all other patient and hospital characteristics, regional variation persisted, with highest odds of transfer with hospitalization in the Northeast, indicating variability by region not explained by other factors, and findings supported by other limited data. ${ }^{31}$ This variability was further elucidated in our examination of change in variance estimates accounting for patient, then hospital, characteristics. Although we expected and found marked variability in hospital transfer rates in our null mod- el (without accounting for any patient or hospital characteristics), we interestingly found that variability increased upon adjusting for patient characteristics. This result is presumably due to the fact that patients who are more likely to be transferred (ie, "sick" patients) are more often already at hospitals less likely to transfer patients, supported by our findings that hospital CMI is inversely associated with odds of transfer (in other words, hospitals that care for a less sick patient population are more likely to transfer their patients, and hospitals that care for a sicker patient population [higher CMI] are less likely to transfer). Adjusting solely for patient characteristics effectively equalizes these patients across hospitals, which would lead to even increased variability in transfer rates. Conversely, when we then adjusted for hospital characteristics, variability in hospital transfer rates decreased by $83 \%$ (in other words, hospital characteristics, rather than patient characteristics, explained much of the variability in transfer rates), although significant unexplained variability remained. We should note that although the observed reduction in variability was explained by the patient and hospital characteristics included in the model, these character- 
TABLE 2. Patient and Hospital Predictors of Transfer

\begin{tabular}{|c|c|c|}
\hline Characteristic & $\begin{array}{l}\text { Adjusted Odds of } \\
\text { Transfer }(95 \% \mathrm{Cl})^{\mathrm{a}}\end{array}$ & $P$ value \\
\hline \multicolumn{3}{|l|}{ Patient Characteristics } \\
\hline \multicolumn{3}{|l|}{ Age, y } \\
\hline 65-74 (referent) & -- & \\
\hline $75-84$ & $2.38(2.33,2.43)$ & $<0.001$ \\
\hline$\geq 85$ & $1.89(1.85,1.93)$ & $<0.001$ \\
\hline \multicolumn{3}{|l|}{ Sex } \\
\hline Male & $1.11(1.09,1.12)$ & $<0.001$ \\
\hline Female (referent) & -- & -- \\
\hline \multicolumn{3}{|l|}{ Race } \\
\hline White & $1.17(1.13,1.20)$ & $<0.001$ \\
\hline Black (referent) & -- & -- \\
\hline Hispanic & $1.16(1.09,1.24)$ & $<0.001$ \\
\hline Other & $1.34(1.28,1.41)$ & $<0.001$ \\
\hline \multicolumn{3}{|l|}{ DRG-weight quartile } \\
\hline Lowest quartile & $2.02(1.95,2.09)$ & $<0.001$ \\
\hline $2^{\text {nd }}$ quartile & $1.85(1.75,1.91)$ & $<0.001$ \\
\hline $3^{\text {rd }}$ quartile & $1.51(1.46,1.55)$ & $<0.001$ \\
\hline Highest quartile (referent) & -- & -- \\
\hline \multicolumn{3}{|c|}{ Primary diagnosis on admission } \\
\hline AMl & $25.1(23.2,27.2)$ & $<0.001$ \\
\hline CHF & $3.76(3.51,4.02)$ & $<0.001$ \\
\hline Sepsis & $2.58(2.41,2.76)$ & $<0.001$ \\
\hline Arrhythmia & $6.18(5.77,6.63)$ & $<0.001$ \\
\hline Stroke & $4.84(4.46,5.26)$ & $<0.001$ \\
\hline Pneumonia & $1.90(1.78,2.04)$ & $<0.001$ \\
\hline Gl bleed & $3.84(3.58,4.13)$ & $<0.001$ \\
\hline Renal failure & $2.31(2.15,2.48)$ & $<0.001$ \\
\hline Esophageal & $1.99(1.86,2.14)$ & $<0.001$ \\
\hline COPD & $1.91(1.74,2.09)$ & $<0.001$ \\
\hline Hip fracture/dislocation & $4.37(4.03,4.75)$ & $<0.001$ \\
\hline Chest pain & $3.53(3.21,3.87)$ & $<0.001$ \\
\hline UTI (referent) & -- & -- \\
\hline Respiratory disease & $1.16(1.08,1.25)$ & $<0.001$ \\
\hline Metabolic & $1.30(1.20,1.41)$ & $<0.001$ \\
\hline Other & $3.89(3.65,4.13)$ & $<0.001$ \\
\hline HCC risk score ${ }^{b}$ & $1.37(1.36,1.37)$ & $<0.001$ \\
\hline
\end{tabular}

Medicaid co-insurance

Yes (referent)

$\begin{array}{lll}\text { No } & 1.50(1.47,1.53) & <0.001\end{array}$

Number of hospitalizations in the past 12 months

\begin{tabular}{|c|c|c|}
\hline 0 & $1.87(1.79,1.95)$ & $<0.001$ \\
\hline 1 & $1.49(1.42,1.55)$ & $<0.001$ \\
\hline $2-3$ & $1.30(1.24,1.36)$ & $<0.001$ \\
\hline$\geq 4$ (referent) & -- & -- \\
\hline \multicolumn{3}{|l|}{ Season of hospital admission ${ }^{c}$} \\
\hline Q1 & $1.04(1.03,1.06)$ & $<0.001$ \\
\hline Q2 & $1.05(1.03,1.07)$ & $<0.001$ \\
\hline Q3 & $1.07(1.05,1.09)$ & $<0.001$ \\
\hline Q4 (referent) & -- & -- \\
\hline Median income per household by census tract, per $\$ 1000$ & $1.00(1.00,1.00)$ & 0.58 \\
\hline \multicolumn{3}{|l|}{ Index Hospital Characteristics ${ }^{d}$} \\
\hline \multicolumn{3}{|l|}{ Size } \\
\hline Small (<99 beds) & $2.30(1.95,2.72)$ & $<0.001$ \\
\hline Medium (100-399 beds) & $1.67(1.44,1.95)$ & $<0.001$ \\
\hline Large (>400 beds)(referent) & -- & -- \\
\hline
\end{tabular}

Continued on page 441 istics do not necessarily justify the variability they accounted for; although patients' race or hospitals' location may explain some of the observed variability, this does not reasonably justify it.

This observed variability in transfer practices is not surprising given the absence of standardization and clear guidelines to direct clinical IHT practice. ${ }^{17}$ Selection of patients that may benefit from transfer is often ambiguous and subjective. ${ }^{6}$ The Emergency Medical Treatment and Active Labor Act laws dictate that hospitals transfer patients requiring a more specialized service, or when "medical benefits ... outweigh the increased risks to the individual...," although in practice this provides little guidance to practitioners. ${ }^{1}$ Thus, clearer guidelines may be necessary to achieve less variable practices.

Our study is subject to several limitations. First, although nationally representative, the Medicare population is not reflective of all hospitalized patients nationwide. Additionally, we excluded patients transferred from the emergency room. Thus, the total number of patients who undergo IHT nationally is expected to be much higher than reflected in our analysis. We also excluded patients who were transferred more than once during a given hospitalization. This enabled us to focus on the initial transfer decision but does not allow us to look at patients who are transferred to a referral center and then transferred back. Second, given the criteria we used to define transfer, it is possible that we included nontransferred patients within our transferred cohort if they were discharged from one hospital and admitted to a different hospital within 1 day. However, on quality assurance analyses where we limited our cohort to only those beneficiaries with corresponding "transfer in" and "transfer out" claims ( $87 \%$ of the total cohort), we found no marked differences in our results. Additionally, although we assume that patient transfer status was coded correctly within the Medicare dataset, we could not confirm by individually examining each patient we defined as "transferred." However, on additional quality assurance analyses where we examined randomly selected excluded patients with greater than 1 transfer during hospitalization, we found differing provider numbers with each transfer, suggesting validity of the coding. Third, because there are likely many unmeasured patient confounders, we cannot be sure how much of the between-hospital variation is due to incomplete adjustment for patient characteristics. However, since adjusting for patient characteristics actually increased variability in hospital transfer rates, it is unlikely that residual patient confounders fully explain our observed results. Despite this, other variables that are not available within the CMS or AHA datasets may further eluci- 
date hospital transfer practices, including variables reflective of the transfer process (eg, time of day of patient transfer, time delay between initiation of transfer and patient arrival at accepting hospital, accepting service on transfer, etc.); other markers of illness severity (eg, clinical service at the time of index admission, acute physiology score, utilization of critical care services on arrival at receiving hospital); and other hospital system variables (ie, membership in an accountable care organization and/or regional care network, the density of nearby tertiary referral centers (indicating possible supply-induced demand), other variables reflective of the "transfer culture" (such as the transfer rate at the hospital or region where the attending physician trained, etc.). Lastly, though our examination provides important foundational information regarding IHT nationally, this study did not examine patient outcomes in transferred and nontransferred patients, which may help to determine which patients benefit (or do not benefit) from transfer and why. Further investigation is needed to study these outcomes.

\section{CONCLUSION}

In this national study of IHT, we found that a sizable number of patients admitted to the hospital undergo transfer to another acute care facility. Patients are transferred with common medical conditions, including those requiring specialized care such as AMI, and a high rate of comorbid clinical conditions, and certain patient and hospital characteristics are associated with greater odds of transfer. Although many of the observed associations between characteristics and odds of transfer were expected based on limited existing literature, we found several unexpected findings, eg, suggesting the possibility of a threshold beyond which sicker patients are not transferred. Additionally, we found that black and Medicaid patients had lower odds of transfer, which warrants further investigation for potential health care disparity. Importantly, we found much variability in the practice of IHT, as evidenced by the inexplicable differences in transfer by hospital region, and by residual unexplained variability in hospital transfer rates after accounting for patient and hospital characteristics, which may be due to lack of standard guidelines to direct IHT practices. In conclusion, this study of hospitalized Medicare patients provides important foundational information regarding rates and predictors of IHT nationally, as well as unexplained variability that exists within this complex care transition. Further investigation will be essential to understand reasons for, processes related to, and outcomes of transferred patients, to help guide standardization in best practices in care.

Disclosure: Nothing to report.
TABLE 2. Patient and Hospital Predictors of Transfer (continued)

Adjusted Odds of

Transfer $(95 \% \mathrm{Cl})^{\mathrm{a}} \quad P$ value

ndex Hospital Characteristics ${ }^{d}$

Geographic location

Northeast $\quad 1.40(1.27,1.55)<0.001$

Midwest $\quad 1.27(1.17,1.39) \quad<0.001$

$\begin{array}{lll}\text { South } & 1.10(1.02,1.19) & 0.02\end{array}$

West (referent)

For-profit (referent)

Not-for-profit $\quad 1.09(1.02,1.18) \quad 0.017$

Public

$1.27(1.17,1.38) \quad<0.001$

aching status

Major (referent)

Minor

Nonteaching

$1.53(1.22,1.92)$

$<0.001$

$1.79(1.42,2.26) \quad<0.001$

ocation

$\begin{array}{lll}\text { Rural } & 1.24(1.15,1.34) & <0.001\end{array}$

CMI $0.36(0.30,0.45) \quad<0.001$

Absence of certified trauma center

$1.04(0.98,1.10) \quad 0.179$

Absence of medical intensive care unit

$1.11(1.00,1.22)$

0.022

bsence of cardiac intensive care unit

$1.07(1.00,1.14)$

0.056

Absence of cardiac surgery services

$2.52(2.28,2.79)$

$<0.001$

Absence of adult interventional cardiac catheterization

$1.21(1.11,1.30)$

$<0.001$

absence of adult cardiac electrophysiology

$1.17(1.09,1.27)$

$<0.001$

0.99(0.98, 1.00)

0.0009

Adjusted for all patient and hospital characteristics, accounting for fixed effects for diagnosis and for the clustering of patients within

(n)

IOTE: Abbreviations: AMl, acute myocardial infarction; CHF, congestive heart failure: $\mathrm{Cl}$, confidence interval: $\mathrm{CMl}$, case mix index; MS, Centers for Medicare and Medicaid Services; COPD, chronic obstructive pulmonary disease; DRG, diagnosis-related group; Gl, gastrointestinal; HCC, Hierarchical Condition Category; SD, standard deviation; UTI, urinary tract infection.

\section{References}

1. Iwashyna TJ. The incomplete infrastructure for interhospital patient transfer. Crit Care Med. 2012;40(8):2470-2478.

2. Iwashyna TJ, Christie JD, Moody J, Kahn JM, Asch DA. The structure of critical care transfer networks. Med Care. 2009;47(7):787-793.

3. Mehta RH, Stalhandske EJ, McCargar PA, Ruane TJ, Eagle KA. Elderly patients at highest risk with acute myocardial infarction are more frequently transferred from community hospitals to tertiary centers: reality or myth? Am Heart J. 1999;138(4 Pt 1):688-695.

4. Iwashyna TJ, Kahn JM, Hayward RA, Nallamothu BK. Interhospital transfers among Medicare beneficiaries admitted for acute myocardial infarction at nonrevascularization hospitals. Circ Cardiovasc Qual Outcomes. 2010;3(5):468-475.

5. Roe MT, Chen AY, Delong ER, Boden WE, Calvin JE Jr, Cairns CB, et al. Patterns of transfer for patients with non-ST-segment elevation acute coronary syndrome from community to tertiary care hospitals. Am Heart J. 2008;156(1):185-192.

6. Bosk EA, Veinot T, Iwashyna TJ. Which patients and where: a qualitative study of patient transfers from community hospitals. Med Care. 2011;49(6):592-598.

7. Wagner J, Iwashyna TJ, Kahn JM. Reasons underlying interhospital transfers to an academic medical intensive care unit. J Crit Care. 2013;28(2):202-208.

8. Cohen MD, Hilligoss PB. The published literature on handoffs in hospitals: deficiencies identified in an extensive review. Qual Saf Health Care. 2010;19(6):493-497.

9. Riesenberg LA, Leitzsch J, Massucci JL, et al. Residents' and attending physicians' handoffs: a systematic review of the literature. Acad Med. 2009;84(12):1775-1787.

10. Arora V, Johnson J, Lovinger D, Humphrey HJ, Meltzer DO. Communication failures in patient sign-out and suggestions for improvement: a critical incident analysis. Qual Saf Health Care. 2005;14(6):401-407.

11. Sokol-Hessner L, White AA, Davis KF, Herzig SJ, Hohmann SF. Interhospital 
transfer patients discharged by academic hospitalists and general internists: characteristics and outcomes. J Hosp Med. 2016;11(4):245-250.

12. Bernard AM, Hayward RA, Rosevear J, Chun H, McMahon LF. Comparing the hospitalizations of transfer and non-transfer patients in an academic medical center. Acad Med. 1996;71(3):262-266.

13. Golestanian E, Scruggs JE, Gangnon RE, Mak RP, Wood KE. Effect of interhospital transfer on resource utilization and outcomes at a tertiary care referral center. Crit Care Med. 2007;35(6):1470-1476.

14. Durairaj L, Will JG, Torner JC, Doebbeling BN. Prognostic factors for mortality following interhospital transfers to the medical intensive care unit of a tertiary referral center. Crit Care Med. 2003;31(7):1981-1986.

15. Kerr HD, Byrd JC. Community hospital transfers to a VA Medical Center. JAMA 1989;262(1):70-73.

16. Dragsted L, Jörgensen J, Jensen $\mathrm{NH}$, et al. Interhospital comparisons of patient outcome from intensive care: importance of lead-time bias. Crit Care Med. 1989;17(5):418-422

17. Gupta K, Mueller SK. Interhospital transfers: the need for standards. J Hosp Med. 2015;10(6):415-417

18. The Dartmouth Atlas of Health Care: Understanding of the Efficiency and Effec tiveness of the Health Care System. The Dartmouth Institute for Health Practice and Clinical Policy, Lebanon, NH. http://www.dartmouthatlas.org/. Accessed November 1,2016

19. American Hospital Association Annual Survey Database. American Hospital Association, Chicago, IL. http://www.ahadataviewer.com/book-cd-products/ AHA-Survey/. Accessed July 1, 2013.

20. U.S. Department of Health and Human Services (HRSA): What are critical access hospitals (CAH)? http:/www.hrsa.gov/healthit/toolbox/RuralHealthITtoolbox/ Introduction/critical.html. Accessed June 9, 2016.

21. Li P, Kim MM, Doshi JA. Comparison of the performance of the CMS Hierar chical Condition Category (CMS-HCC) risk adjuster with the Charlson and Elixhauser comorbidity measures in predicting mortality. BMC Health Serv Res. 2010;10:245

22. Hernandez-Boussard T, Davies S, McDonald K, Wang NE. Interhospital facility transfers in the United States: a nationwide outcomes study. J Patient Saf. Nov 132014.

23. Landon BE, Normand SL, Lessler A, et al. Quality of care for the treatment of acute medical conditions in US hospitals. Arch Intern Med. 2006;166(22): 2511-2517.

24. Mueller SK, Lipsitz S, Hicks LS. Impact of hospital teaching intensity on quality of care and patient outcomes. Med Care.2013;51(7):567-574.

25. Lopez L, Hicks LS, Cohen AP, McKean S, Weissman JS. Hospitalists and the quality of care in hospitals. Arch Intern Med. 2009;169(15):1389-1394.

26. Barreto-Filho JA, Wang Y, Rathore SS, et al. Transfer rates from nonprocedure hospitals after initial admission and outcomes among elderly patients with acute myocardial infarction. JAMA Intern Med. 2014;174(2):213-222.

27. Carlson JE, Zocchi KA, Bettencourt DM, et al. Measuring frailty in the hospitalized elderly: concept of functional homeostasis. Am J Phys Med Rehabil. 1998;77(3):252-257.

28. Ward E, Jemal A, Cokkinides V, et al. Cancer disparities by race/ethnicity and socioeconomic status. CA Cancer J Clin. 2004;54(2):78-93.

29. Iribarren C, Tolstykh I, Somkin CP, et al. Sex and racial/ethnic disparities in outcomes after acute myocardial infarction: a cohort study among members of a large integrated health care delivery system in northern California. Arch Intern Med. 2005;165(18):2105-2113.

30. Kawachi I, Daniels N, Robinson DE. Health disparities by race and class: why both matter. Health Aff (Millwood). 2005;24(2):343-352.

31. Herrigel DJ, Carroll M, Fanning C, Steinberg MB, Parikh A, Usher M. Interhospital transfer handoff practices among US tertiary care centers: a descriptive survey. J Hosp Med. 2016;11(6):413-417. 